\title{
Electrochemical detection of ascorbic acid in artificial sweat using a flexible alginate/CuO-modified electrode
}

\author{
Bergoi Ibarlucea $^{1,2}$ (D) - Arnau Pérez Roig ${ }^{1} \cdot$ Dmitry Belyaev $^{1} \cdot$ Larysa Baraban $^{1,2} \cdot$ Gianaurelio Cuniberti $^{1,2}$
}

Received: 16 May 2020 / Accepted: 18 August 2020 / Published online: 27 August 2020

(C) The Author(s) 2020

\begin{abstract}
A flexible sensor is presented for electrochemical detection of ascorbic acid in sweat based on single-step modified gold microelectrodes. The modification consists of electrodeposition of alginate membrane with trapped $\mathrm{CuO}$ nanoparticles. The electrodes are fabricated at a thin polyimide support and the soft nature of the membrane can withstand mechanical stress beyond requirements for skin monitoring. After characterization of the membrane via optical and scanning electron microscopy and cyclic voltammetry, the oxidative properties of $\mathrm{CuO}$ are exploited toward ascorbic acid for amperometric measurement at micromolar levels in neutral buffer and acidic artificial sweat, at ultralow applied potential $(-5 \mathrm{mV}$ vs. Au pseudo-reference electrode). Alternatively, measurement of the horizontal shift of redox peaks by cyclic voltammetry is also possible. Obtaining a limit of detection of $1.97 \mu \mathrm{M}$, sensitivity of $0.103 \mathrm{~V} \log (\mu \mathrm{M})^{-1}$ of peak shift, and linear range of $10-150 \mu \mathrm{M}$, the effect of possible interfering species present in sweat is minimized, with no observable cross-reaction, thus maintaining a high degree of selectivity despite the absence of enzymes in the fabrication scheme. With a lateral flow approach for sample delivery, repeated measurements show recovery in few seconds, with relative standard deviation of about $20 \%$, which can serve to detect increased loss or absence of vitamin, and yet be improved in future by optimized device designs. This sensor is envisioned as a promising component of wearable devices for e.g. non-invasive monitoring of micronutrient loss through sweat, comprising features of light weight, low cost, and easy fabrication needed for such application.
\end{abstract}

Keywords Ascorbic acid $\cdot$ Nonenzymatic sensor $\cdot$ Electroanalysis $\cdot \mathrm{CuO}$ nanoparticles

\section{Introduction}

The long-time known essential ascorbic acid (vitamin C) is a water-soluble molecule that, like other water-soluble vitamins, cannot be stored in the body for a long time [1]; it can be found

Electronic supplementary material The online version of this article (https://doi.org/10.1007/s00604-020-04510-5) contains supplementary material, which is available to authorized users.

Bergoi Ibarlucea

bergoi.ibarlucea@tu-dresden.de

Larysa Baraban

1.baraban@hzdr.de

1 Institute for Materials Science and Max Bergmann Center for Biomaterials, Technische Universität Dresden, Dresden, Germany

2 Center for advancing electronics Dresden (cfaed), Technische Universität Dresden, Dresden, Germany

3 Present address: Institute of Radiopharmaceutical Cancer Research, Helmholtz-Zentrum Dresden-Rossendorf e.V., Dresden, Germany in secreted body fluids, e.g., sweat [2]. Consequently, heatexposed activities (e.g., in steel factories) or prolonged sport sessions result in nutrient loss through sweating, including that of ascorbic acid [3], making it necessary to monitor them for the on-time response by dietary intake without delay. Thus, ascorbic acid is an important target for quantitative sensing and monitoring in the biomedical field, relevant also for the food and beverage industry, e.g., for quality control. It is typically measured by high-performance liquid chromatography [4], enzyme-based colorimetric multiwell kits [5], or capillary electrophoresis [6], with limits of detection and linear ranges oscillating between nanomolar and micromolar concentrations. These methods are remote laboratory techniques, with no possibility for on-site measurements.

Taking into account modern day lifestyle and needs, the sensor design principles should evolve correspondingly [7]. In special professional groups like athletes or heat-exposed workers, a miniaturized electrical device with low power requirements and low cost but retaining high sensitivity, integrated in the garment or the skin, would fulfill the 
requirements of a continuous monitoring for preventive healthcare application. In this context, micro- and nanofabrication techniques play an important role by helping to perform detection processes in a small and low-cost chip [8, 9] with the potential to include flexible characteristics to withstand mechanical stress produced by the movement of the end user [10-13]. Several advanced examples of flexible and even stretchable sensors that could potentially be applied for sweat sensing can be found in the state of the art, based on different materials and focused on different target analytes. The most usual materials that can be found are polymeric, like polydimethylsiloxane, with channels containing dyes for the colorimetric sensing of $\mathrm{pH}$, lactate, glucose, and chloride [14], or polyethylene terephthalate, with electrodes modified with the corresponding enzymes and ionophores for electrochemical and potentiometric detection of glucose, lactate, sodium, and potassium [13]. Other materials can be found as well, like temporary transfer tattoo paper modified with enzymemodified electrodes for lactate detection [15], or conductive textiles modified with iridium oxide for $\mathrm{pH}$ monitoring [16]. Preferably, the fabrication process should be as simple as possible in order to allow a cheap and rapid workflow. Some recent examples of such rapid fabrication include galvanostatic electrodeposition of hydrogels containing enzymes [17], stamping of electrode material with further simple enzyme cross-linking [18], or material vacuum filtration through a mask [19] followed by layer-by-layer or potentiodynamic deposition of receptor layers. Similar concepts would be desired for ascorbic acid measurement.

Most of the recently reported biosensors for ascorbic acid are electrochemical, either enzymatic or nonenzymatic, as it will be described in the following lines. The first group makes use of the natural selectivity of enzymes to discriminate between analyte and possible interferents, being ascorbate oxidase the one used for the present case [20]. This enzyme catalyzes the oxidation of ascorbic acid to dehydroascorbic acid by accepting electrons on its copper center, causing the electron movement that can be measured by the transducer, as it was recently shown with an electrochemical tattoo with an integrated iontophoresis structure to extract sweat [21]. On the other side, nonenzymatic sensors make use of new materials to overcome the limitations of biological receptors in terms of cost and stability [22]. A multitude of attempts have pursued this approach for ascorbic acid determination, for example using carbon-supported PdNi nanoparticles (NPs) [23], carbon nanoplatelets derived from ground cherry husks [24], silver NPs grafted graphene/polyaniline nanocomposites [25], molybdophosphate films [26], graphene oxide/multi-walled carbon nanotubes/gold nanorods combinations [27], ZiNi nanoalloy-modified carbon nanotubes [28], or $\mathrm{TiO}_{2} /$ reduced graphene oxide nanocomposites [29]. Some sensors provide an added value with the possibility of performing the simultaneous detection of various analytes that typically show overlapping oxidation potentials [30-32]. Krishnan et al. [33] proposed indium tin oxide electrodes with graphitic $\mathrm{C}_{3} \mathrm{~N}_{4}$ nanosheets that were modified with nanocomposites made of mesoporous Ag-doped $\mathrm{TiO}_{2}-\mathrm{SnO}_{2}$. Differential pulsed voltammetry experiments allowed to obtain three distinct peaks for ascorbic acid, dopamine, and uric acid in 50fold diluted urine. The same three analytes can be distinguished as well by using combinations of different materials, among which we may mention: $\mathrm{TmPO}_{4}$ and graphene oxide composites [34], assemblies of positively charged Zn-NiAllayered double hydroxide with negatively charged reduced graphene oxide [35], or three dimensional porous structures of $\mathrm{MoS}_{2}$ and reduced graphene oxide composites [36]. Instead, if reduced graphene oxide containing $\mathrm{Fe}_{3} \mathrm{O}_{4}$ nanoparticles is modified with hydroxypropyl- $\beta$-cyclodextrin, ascorbic acid, serotonin, and dopamine can be distinguished using the same electrochemical technique [37], as demonstrated in serum samples. On the other hand, it is also known that metal oxide NPs provide a high surface area and good electron transport kinetics [38], which also make them a good candidate for the development of nonenzymatic sensors. Ascorbic acid is known to be a reducing agent for nanostructured copper, being used for NP preparation [39], and as aforementioned, it has a preference for electron donation to the copper center of the ascorbate oxidase enzyme. This property can be exploited for its determination using simpler copper-based nonenzymatic materials, as demonstrated using $\mathrm{CuO}$ (copper oxide) nanowires synthesized on $\mathrm{Cu}$ foils by a thermal oxidation process at $350^{\circ} \mathrm{C}$ for $100 \mathrm{~min}$ [40], or with 3D graphene/ $\mathrm{CuO}$ nanoflowers fabricated by copper electrodeposition on a graphene structure grown by chemical vapor deposition (CVD) on a nickel foam that was later etched [41].

The generality of the aforementioned examples makes use of either high fabrication temperatures, complex equipment (CVD), long fabrication times, or commercially unavailable NPs or nanostructures and nanocomposites that need to be synthesized. In most occasions, the mechanical properties for application in flexible sensors were not tested and in some cases they also result in sensors that measure at a high potential with the resulting increased probability of oxidizing other molecules. Considering the envisioned application of a wearable device for ascorbic acid monitoring in sweat in heatexposed individuals, minimizing the consumed power (and therefore the working potential) is a must together with good mechanical properties (flexible nature) and ease of fabrication in terms of cost and time. The function in acidic solutions should also be demonstrated.

Here, we report on a new ascorbic acid sensor that combines for the first time the rapid fabrication possibility of the alginate membrane with the encapsulation of the commercially available $\mathrm{CuO}$ NPs to exploit their ascorbic acid oxidizing capabilities. The sensor is fabricated via electrodeposition of an alginate membrane using a precursor mixed with $\mathrm{CuO}$ NPs 
on gold electrodes evaporated on a light-weight polyimide support [42]. The alginate membrane, which is reported to be biocompatible and permeable to the diffusion of the electrochemical substrates but impermeable to cells or large particles, has previously been used to trap enzymes for glucose and lactate determination [17, 43] and carbon nanotubes to measure microbial activity [44]. Here, we trap CuO NPs from a commercially available and low-cost nanopowder to make use of their natural interaction with ascorbic acid previously mentioned. The membrane is formed in few minutes in one step and its size can be controlled by the electrodeposition rate [45]. Moreover, it can be easily removed by using a calcium chelating buffer like phosphate-buffered saline (PBS), offering reusability of the electrodes to immobilize a new membrane with possibly different catalysts for additional measurements [17]. The use of simple gold electrodes on polyimide makes it compatible with a variety of techniques like inkjet printing or photolithography and with the future application as a flexible, wearable device. Finally, the determination of ascorbic acid is done at the micromolar range as it is found in sweat [3,46], and in artificial perspiration solution without interference of any of the other present biomolecules.

\section{Materials and methods}

\section{Materials and reagents}

Support for electrode fabrication: Kapton HN500 (125- $\mu \mathrm{m}-$ thick polyimide film from Dupont, USA, www.dupont.com). Chemicals and reagents: alginic acid sodium salt, calcium carbonate (>99\%), $\mathrm{CuO}$ nanopowder $(<50 \mathrm{~nm}), \mathrm{NaCl}(>$ $99.5 \%$ ), imidazole (>99\%), $\mathrm{KCl}(>99 \%)$, and $\mathrm{CaCl}_{2}(>$ $98 \%), \mathrm{NH}_{4} \mathrm{Cl}(>99.5 \%)$, acetic acid $(>99.7 \%)$, lactic acid (sodium L-lactate $>99 \%)$, D(+)-glucose monohydrate (> 99\%), uric acid sodium salt, pyruvic acid sodium salt, Lglutamic acid (>99\%), and urea (>98\%), hexamethyldisilazane (HMDS, $>99 \%$ ) were purchased from Sigma-Aldrich - Germany (www.sigmaaldrich.com). Filter paper for lateral flow sample delivery: 413-type, VWR, Germany, de.vwr.com/store

\section{Electrode fabrication and modification}

Gold electrodes were first fabricated on a $125-\mu \mathrm{m}$-thick polyimide film (Dupont, USA, www.dupont.com) by a standard photolithography process [11] followed by $100 \mathrm{~nm}$ gold thermal evaporation on $3 \mathrm{~nm}$ chromium adhesion layer. The chip (Fig. 1a) consisted of three electrodes, using the smallest one $\left(1.2 \mathrm{~mm}^{2}\right)$ as working electrode, so that the kinetics at the counter electrode do not limit those at the working one. The area of counter and reference electrodes were 3.8 and 4 . $1 \mathrm{~mm}^{2}$, respectively.
The alginate membrane precursor solution was composed by $1 \mathrm{wt} \%$ alginic acid sodium salt and $0.5 \mathrm{wt} \%$ calcium carbonate. A total of $16 \mathrm{mg} \mathrm{mL}^{-1} \mathrm{CuO}$ nanopowder $(<50 \mathrm{~nm})$ was added to the mix as catalyst for the ascorbic acid determination, unless otherwise stated. Twenty microliters of the mix was drop casted on the electrodes (minimum volume to cover reliably the electrodes) and a galvanostatic deposition was carried out using a PalmSens 4 potentiostat-galvanostat at a current density of $1.7 \mathrm{~A} \mathrm{~m}^{-2}$ (larger current densities led to electrode delamination) for $140 \mathrm{~s}$ (until observing stability of the measured potential). The same system was used for all next cyclic voltammetries and chronoamperometries with DC current. The anodic current produces a water electrolysis generating protons that react with the calcium carbonate, which tends to buffer the solution by consuming the protons and forming $\mathrm{Ca}^{2+}$ ions and $\mathrm{CO}_{2}$ [45]. The calcium ions interact with the alginate polymers by cross-linking them and creating the membrane, where the CuO NPs get trapped (Fig. 1ai). The process resulted in a flexible chip with a membrane on the working electrode (Fig. 1a-ii). The drop with the precursor mix could be recovered and used for the deposition on further electrodes. Subsequently, the formed membrane was rinsed in $145 \mathrm{mM} \mathrm{NaCl}$ and $10 \mathrm{mM}$ imidazole buffer supplemented with $145 \mathrm{mM} \mathrm{KCl}$ to mimic physiological conditions and $10 \mathrm{mM} \mathrm{CaCl}_{2}$ to prevent $\mathrm{Ca}^{2+}$ ion loss and therefore membrane dissolution. This imidazole buffer, adjusted to $\mathrm{pH}$ 7.4, was used for the electrical characterization of the sensor as well as for ascorbic acid measurements in buffer and at room temperature.

\section{Alginate membrane characterization}

The membrane was characterized by optical microscopy, scanning electron microscopy (SEM), and cyclic voltammetry.

Larger current densities than $1.7 \mathrm{~A} \mathrm{~m}^{-2}$ led to electrode delamination, while smaller ones produced weak and unstable membranes that are easily removed. Shorter electrodeposition times $(60 \mathrm{~s})$ resulted in soft membranes from which the CuO NPs were easily washed away (Fig. S1, Supplementary Material), while above $140 \mathrm{~s}$ the membrane was adsorbed to the electrode in a stable manner during the rinsing steps and the NPs covered the whole working electrode. The evolution of the potential drift due to the galvanostatic deposition process is shown in Fig. S2, where stability starts to be observed after one minute. The resulting membrane after $140 \mathrm{~s}$ had a thickness of ca. $10 \mu \mathrm{m}$ as measured via digital microscopy (Fig. 1a, iv) with a toluidine blue $\mathrm{O}$ stained membrane to confer a more homogeneous and non-particulated contrast.

The membranes were prepared for SEM observation following a protocol for hydrogel fixation reported elsewhere [47] but replacing the used buffer for the imidazole-based 


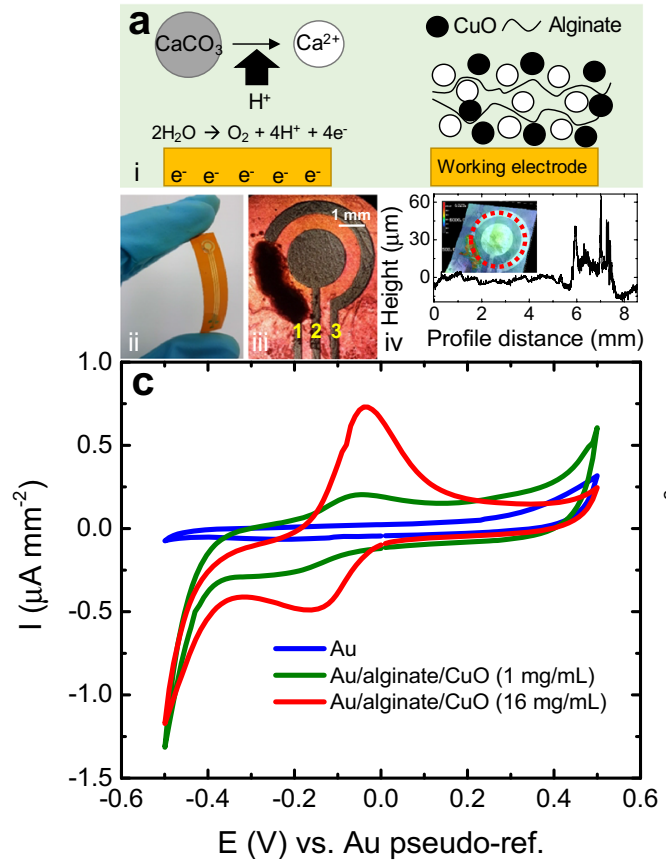

Fig. 1 (a) Alginate electrodeposition with $\mathrm{CuO}$ nanopowder. (i) Scheme of electrodeposition mechanism. The flexible chip is shown in (ii), with the membrane formed after $140 \mathrm{~s}$ electrodeposition time. Yellow numbers indicate electrode function: (1) working, (2) counter, (3) pseudo-reference. Film thickness $(10 \mu \mathrm{m})$ is shown in (iv), measured along the red dotted circle in inset. (b) SEM imaging of alginate membrane with $\mathrm{CuO}$ NPs. Backscattered electrons provide the contrast, with the white spots

one to avoid membrane dissolution. First, hydrogels with and without $\mathrm{CuO}$ NPs were fixed in $2.5 \%$ glutaraldehyde in imidazole buffer for $2 \mathrm{~h}$ at room temperature. They were then left at $4{ }^{\circ} \mathrm{C}$ for $12 \mathrm{~h}$. After washing twice for $5 \mathrm{~min}$ in imidazole buffer, the samples were dehydrated in solutions of increasing ethanol concentration $(10,25,50,70,90$, and $100 \%)$ for $5 \mathrm{~min}$ per concentration. Later, the alginate hydrogel was incubated for $20 \mathrm{~min}$ in 50\% HMDS diluted in ethanol followed by an additional incubation in a new solution with same characteristics. Finally, the double incubation was repeated in two $100 \%$ HMDS solutions. Membranes were left to dry overnight in a desiccator and coated with a $20-\mathrm{nm}$ Au layer by thermal evaporation.

\section{Bending test}

The flexibility of the sensor owing to the mechanical properties of the thin polyimide foil and the soft nature of the alginate was tested by performing cyclic voltammetries before and after controlled bending events. For this, the chip with the electrodeposited membrane was placed on a cylinder of 5-mm radius and bent 1000 times in 10 cycles of 100 bending events each. Cyclic voltammetries were performed in imidazole buffer

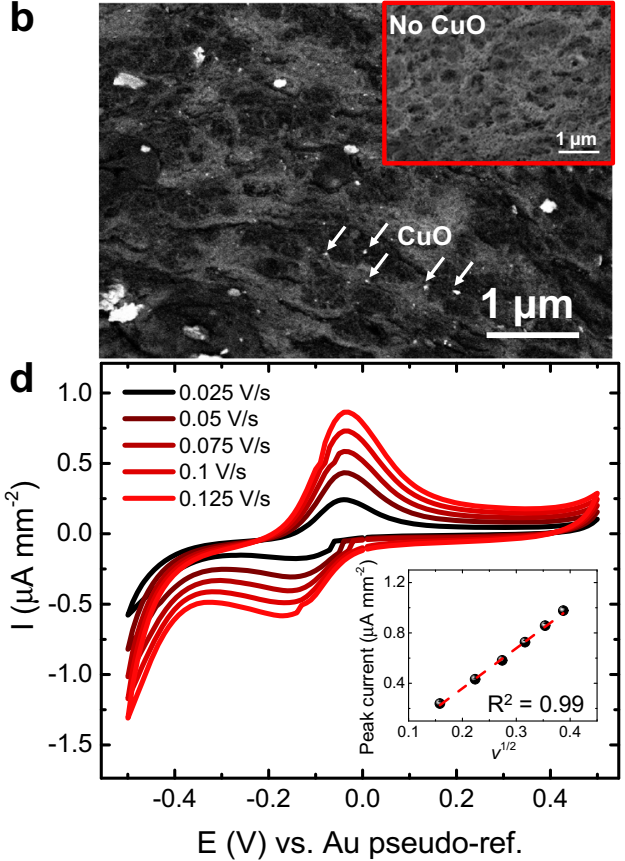

belonging to the $\mathrm{CuO}$ (arrows indicate some examples). Inset shows the membrane without $\mathrm{CuO}$, where no white spots can be seen. (c) Cyclic voltammogram of bare electrodes (blue line) and modified electrodes (red and green), showing the appearance of the redox pair belonging to $\mathrm{CuO}$. (d) Scan rate test and calibration (inset) showing linear relation with square root of the scan rate. All cyclic voltammetries were performed in imidazole buffer at $\mathrm{pH} 7.4$ and room temperature

( $\mathrm{pH} 7.4$, room temperature) between each cycle to observe variations in the signal.

\section{Ascorbic acid determination}

All measurements in buffer were done in the aforementioned imidazole system as non-chelating buffer, adjusted to $\mathrm{pH} 7.4$ and at room temperature. First, cyclic voltammetry at $0.1 \mathrm{~V} \mathrm{~s}^{-1}$ was used to observe the effect on the signal produced by the effect of ascorbic acid. Then, the optimal working potential was chosen for amperometric measurements with different ascorbic acid concentrations at constant $\mathrm{pH}$, to calibrate the sensor and determine the concentration range that could be measured. Next, we proceeded to measure in artificial sweat samples. For this, an artificial perspiration solution was prepared based on the work by Kilic et al. [46], which was composed of $20 \mathrm{~g} \mathrm{~L}^{-1} \mathrm{NaCl}, 17.5 \mathrm{~g} \mathrm{~L}^{-1} \mathrm{NH}_{4} \mathrm{Cl}, 5 \mathrm{~g} \mathrm{~L}^{-1}$ acetic acid, $15 \mathrm{~g} \mathrm{~L}^{-1}$ lactic acid, $0.17 \mathrm{mM}$ glucose, $59 \mathrm{uM}$ uric acid, $0.18 \mathrm{mM}$ pyruvic acid, $0.37 \mathrm{mM}$ glutamic acid, and $10 \mathrm{mM}$ urea. As sweat is a mildly acidic fluid [48, 49], the $\mathrm{pH}$ was adjusted to $\mathrm{pH} 5.5$ using $\mathrm{HCl}$. Prior to the measurements directly in this solution, the possible interference by the presence of the organic molecules in the recipe was analyzed. Finally, the artificial perspiration solution was spiked with ascorbic acid and measured in the same way. Considering the 
difficulties of delivering sweat from skin to a wearable device, we also propose a lateral flow approach as a preliminary design by placing a rectangular filter paper (413-type, VWR, Germany, de.vwr.com/store) in perpendicular to the sensor, and directly drop casting $10 \mu \mathrm{L}$ samples of the artificial sweat with $100 \mu \mathrm{M}$ ascorbic acid which were absorbed and redirected toward the sensing area.

\section{Results and discussion}

\section{Sensor characterization}

SEM imaging of backscattered electrons showed the contrast between the membrane material and the trapped $\mathrm{CuO}$ (Fig. 1b). The porous membrane appears dark, with white spots belonging to the $\mathrm{CuO}$ nanoparticles and aggregates of heterogeneous sizes. In absence of $\mathrm{CuO}$ NPs (inset to Fig. 1b), no white spots could be found. An energy-dispersive X-ray spectroscopy found the presence of carbon and oxygen atoms through the whole surface as part of the alginate membrane, as well as calcium cross-linking the alginic acid (Fig. S3). Carbon only lost its presence in the area where the $\mathrm{CuO}$ particles agglomerated. On these agglomerates, both copper and oxygen were found. The presence of gold atoms could be seen beneath the membrane, as part of the electrodes. The presence of potassium and silicon atoms can be explained as remaining traces from the prior immersions in the imidazole buffer containing $\mathrm{KCl}$ during the rinsing steps, and from the immersion in HMDS in the SEM preparation protocol, respectively.

A cyclic voltammetry of the resulting $\mathrm{CuO}$-containing membrane showed the peaks of the redox pair corresponding to the presence of the $\mathrm{CuO}$ NPs (Fig. 1c). The scan rate test between 25 and $125 \mathrm{mV} \mathrm{s}^{-1}$ showed a linear increase $\left(R^{2}=\right.$ 0.99 , see Fig. S4a for three independent sensors) of the peak amplitude with the square root of the scan rate and with no peak shift (Fig. 1d), indicating a reversible and diffusion controlled system. A slight negative shift was observed only in the cathodic peak; hence, we cannot discard to a certain degree a kinetically controlled behavior [50].

\section{Bending test}

The measured cyclic voltammograms (Fig. 2a) did not show any significant horizontal shift of the redox peak position, which is the effect that allows measuring ascorbic acid in this work. The amplitude of the anodic peak (Fig. 2b) suffered a slight decrease of $1 \mu \mathrm{A}$ during the first 300 bending events and remained constant for the next 700 bends. The amplitude decrease for the cathodic peak instead, decreased less than $0.5 \mu \mathrm{A}$, showing a high performance even after such high deformation. It must be noted that $5 \mathrm{~mm}$ is far beyond the required radius for skin biomonitoring [51].

\section{Ascorbic acid determination in buffer}

Ascorbate produced a pronounced shift of the redox peaks to a positive potential direction (Fig. 3a), indicating a migration of the $\mathrm{CuO}$ through different oxidation states. The linearity with the square root of the scan rate however was not lost (Fig. S4b). Taking the position of the cathodic peak as a reference, the shift increased linearly with the logarithm of the ascorbic acid (Fig. 3b) in a range between 1 and $125 \mu \mathrm{M}$, obtaining a limit of detection (LOD) of $1.97 \pm 1.03 \mu \mathrm{M}$ (calculated as the concentration giving a signal 3-fold the standard deviation of the blank) and a sensitivity $(S)$ of $0.103 \pm 0.004 \mathrm{~V} \log (\mu \mathrm{M})^{-1}$ $\left(y=(0.32 \pm 0.01)+(0103 \pm 0.004) x ; R^{2}=0.99\right)$. As seen from the averaged results of four independent sensors, all of them showed the same measurable range.

The fact that the current measured at zero volts in the backwards sweep of the cyclic voltammetry belongs solely to the capacitive current makes it possible to set this potential in a chronoamperometry and measure the shift of the reducing peak as an increasing current as it shifts and approaches the working potential. Therefore, a chronoamperometry was performed at nearly zero volts ( $-5 \mathrm{mV}$ vs. Au pseudo-ref. electrode), minimizing the possibility to oxidize other possible interfering biomolecules in a real sample. It is important to note that the measurement is also possible at $0 \mathrm{~V}$; however, $5 \mathrm{mV}$ was chosen for the sake of stability of the electrical signal. Imidazole buffer containing ascorbic acid in a range between 1.8 and $125 \mu \mathrm{M}$ was measured. Twenty-microliter samples were drop casted on the sensor and the chronoamperometry was carried out for $60 \mathrm{~s}$ for each concentration, observing an increasing negative current together with the increase in analyte concentration (inset to Fig. 3c). The linear range was observed to be up to $30 \mu \mathrm{M}$ (Fig. 3c, $y=(0.001 \pm 0.002)+(0.0027 \pm$ $\left.\left.2.8 \times 10^{-4}\right) x ; R^{2}=0.95\right)$, allowing to perform measurements in the range where ascorbic acid is found in sweat $(10-30 \mu \mathrm{M})[3,46]$. The LOD in this case was $2.3 \pm 0.2 \mu \mathrm{M}$ (calculated using the $3 \sigma / m$ criterion where $\sigma$ is the standard deviation of the intercept and $m$ is the slope of the calibration plot) and the sensitivity 0.0027 $\pm 2.8 \times 10^{-4} \mu \mathrm{A} \mu \mathrm{M}^{-1} \mathrm{~mm}^{-2}$.

A test with three independent sensors using $6 \mathrm{mg} \mathrm{mL}^{-1}$ $\mathrm{CuO}$ showed, on one hand, that the measurable range was constant and comparable with that obtained using $16 \mathrm{mg} \mathrm{mL}^{-1}$ due to the fact that $\mathrm{CuO}$ is always in molar excess (Fig. S5). However, further reduction to $1 \mathrm{mg} \mathrm{mL}-1$ (voltammogram at Fig. 1c, green line) led to nonreproducible results with highly variable measurable range. We hypothesize that this is caused by the weak redox peaks 


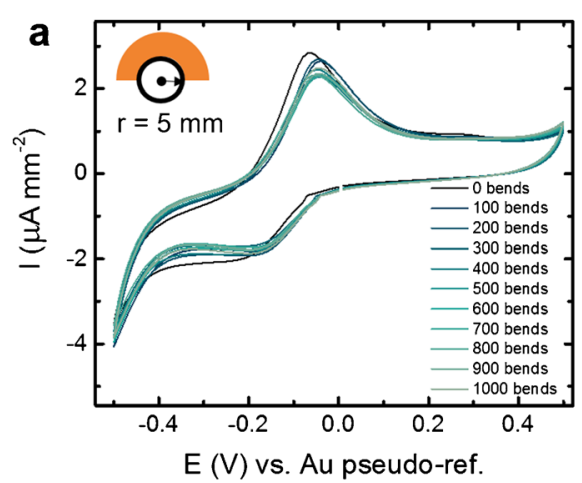

Fig. 2 Results of the bending test at $5 \mathrm{~mm}$-radius in imidazole buffer ( $\mathrm{pH}$ 7.4, room temperature). (a) Cyclic voltammograms and (b) redox peak current density and position before and after each bending cycle. Peak position and current tend to remain around the same values. After an

at low $\mathrm{CuO}$ concentration. Therefore, we can conclude that as long as the redox peaks are clearly visible, the $\mathrm{CuO}$ concentration is not very relevant, at least starting from $6 \mathrm{mg} \mathrm{mL}^{-1}$, concentration that can be chosen as a minimum to measure ascorbic acid reliably, by saving material compared with $16 \mathrm{mg} \mathrm{mL}^{-1}$. With measurements of real samples in mind, we performed an interference test by repeating the chronoamperometry in presence of several biomolecules typically found in sweat: lactic acid, uric acid, pyruvic acid, glutamic acid, glucose, and urea. Twenty microliters of buffer was drop casted on the sensor and the measurement was

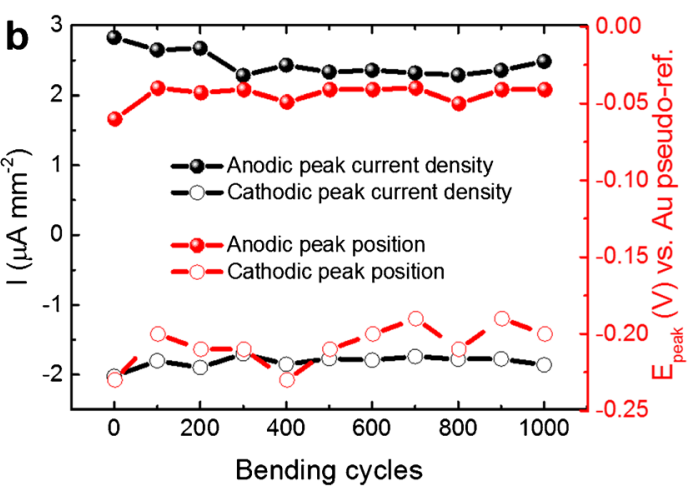

amplitude decrease of less than $1 \mu \mathrm{A}$ during the first 300 bends, it remained constant for the next 700 events. Inset in (a) shows the principle of the test. The orange film represents the chip on polyimide material, bent onto a 5-mm radius

started. After $50 \mathrm{~s}$, additional $20 \mu \mathrm{L}$ buffer was drop casted with each of the biomolecules, reaching the final concentration found in the artificial perspiration solution to be used later on, including various acids (lactic, uric, pyruvic, and glutamic). The results are shown in Fig. 3d. No response was found for any other than ascorbic acid, being this the smallest concentrated one $(10 \mu \mathrm{M})$. Neither did the highly concentrated species at $10 \mathrm{mM}$ (lactic acid, glucose, urea). The response for the ascorbic acid was observed instantaneously, showing the fast response of the sensor.
Fig. 3 Ascorbic acid determination and analysis of the effect of the interferents in imidazole buffer ( $\mathrm{pH} 7.4$, room temperature). (a) Effect of the ascorbic acid on the cyclic voltammogram. The peaks of the $\mathrm{CuO}$ redox pairs are shifted toward positive potential. (b) Averaged calibration of the cathodic peak potential shift of 4 independent sensors (error bars as standard deviation of $n=4$ sensors). (c) Calibration of the amperometry at $-5 \mathrm{mV}$ vs. Au pseudo-ref. electrode in a range between 0 and $125 \mu \mathrm{M}$ (smallest detected concentration $=1.8 \mu \mathrm{M}$ ). Inset shows the real-time measurements. Further repetitions $(n=3)$ shown in Fig. S5. (d) Interference test showing no response with other than ascorbic acid. The arrow indicates the interferent injection moment a
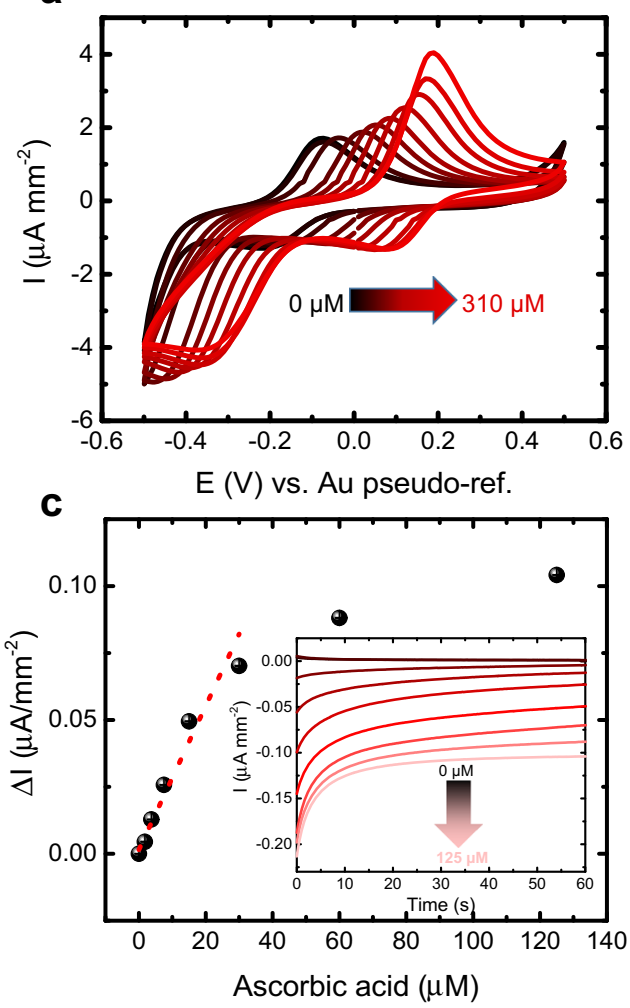

b

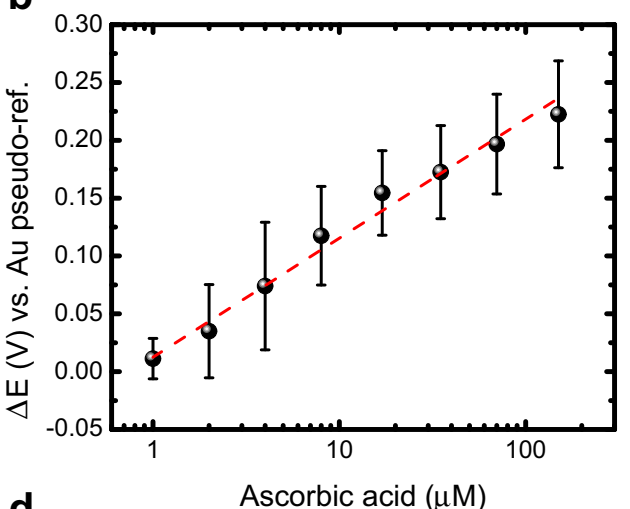

d

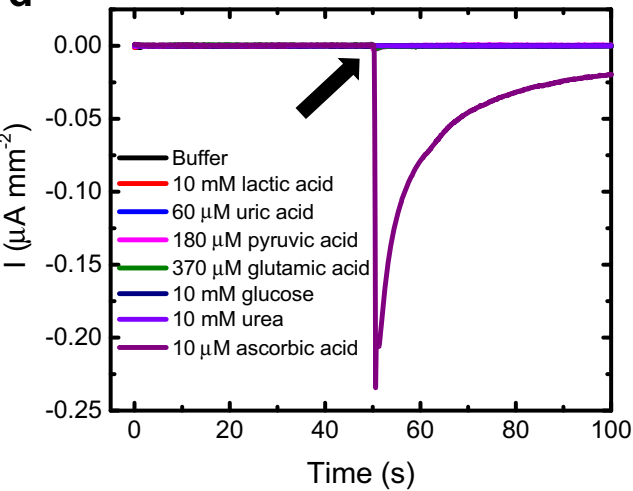




\section{Ascorbic acid determination in artificial samples}

Artificial perspiration solutions were prepared with increasing ascorbic acid concentrations and the chronoamperometry was performed at $-5 \mathrm{mV}$ vs. Au pseudo-ref. electrode. Compared with measurements in imidazole, here the measurable range was extended to higher concentrations, with saturation after ca. $300 \mu \mathrm{M}$ (Fig. 4a, b), which would allow determining the levels found also in blood or a possible excess of ascorbic acid loss through sweat. The current levels were also increased, which is likely coming from the highly complex nature of the solution. The cyclic voltammograms shown in Fig. S6a, $\mathrm{SM}$, support this idea, where the redox peaks and the capacitive current were found to be 4-fold and 2-fold stronger than in imidazole respectively. In spite of that, the levels found in sweat could also be detected, as seen in the real-time measurement of $10 \mu \mathrm{M}$ ascorbic acid (Fig. 4c). By performing the complete cyclic voltammetry for each ascorbate concentration, the quantification should also be possible by measuring the peak shift (Fig. S6b, SM). Furthermore, one hour incubation in sweat did not produce any degradation of the signal (Fig. S7, SM), demonstrating good stability.

Finally, taking into account the complexity of sweat delivery on a wearable device, we propose the combination of the sensor with a lateral flow approach [52], where a hydrophilic paper allows the gradual passing of a liquid sample. As preliminary test, a rectangular piece of filter paper (413-type filter paper, VWR, Germany, de.vwr.com/store) was directly placed on top of the sensor and $10 \mu \mathrm{L}$ samples of artificial sweat with $100 \mu \mathrm{M}$ ascorbic acid was dropped on one side of the paper (inset to Fig. 4d), which absorbed and redirected it toward the sensor, forming clear signal peaks while no signal was observed for the samples without ascorbic acid (Fig. 4d).

Despite the possibility of measuring in the levels and physiological conditions in serum or blood, incubations in fetal bovine serum led to gradual degradation and disappearing of the redox peaks. A previously reported work with $\mathrm{CuO}$ entrapped in alginate together with glucose oxidase for glucose sensing however showed successful glucose determination without $\mathrm{CuO}$ degradation and ascorbic acid interference, which denotes that the presence of the enzyme might play a role in NP protection. In our case, a direct interaction between $\mathrm{CuO}$ and glucose is discarded in the degradation process, as demonstrated in the interference test and the measurements in artificial perspiration solution containing solution, and considering that such interaction requires alkaline conditions [53].

Other nonenzymatic sensors exist with good response at the required micromolar concentrations. A comparison with our work is shown in Table 1. Although many reported works show detection at low potential, ours remains as the one closest to zero volts, and although it was achieved using Au pseudo-ref. electrode, it would definitely help to save battery in a wearable device. The flexible nature of the sensor was also demonstrated and the fabrication was also the simplest, with
Fig. 4 Measurements in artificial perspiration solution, $\mathrm{pH} 5.5$, room temperature. (a)

Chronoamperometry and (b) calibration at $-5 \mathrm{mV}$ vs. Au pseudoref. electrode. Inset shows magnification of the smallest concentrations. (c) Real-time measurement of $10 \mu \mathrm{M}$ ascorbic acid (AA) by direct drop casting. (d) Real-time measurement of sweat with and without ascorbic acid by sample delivery through filter paper a
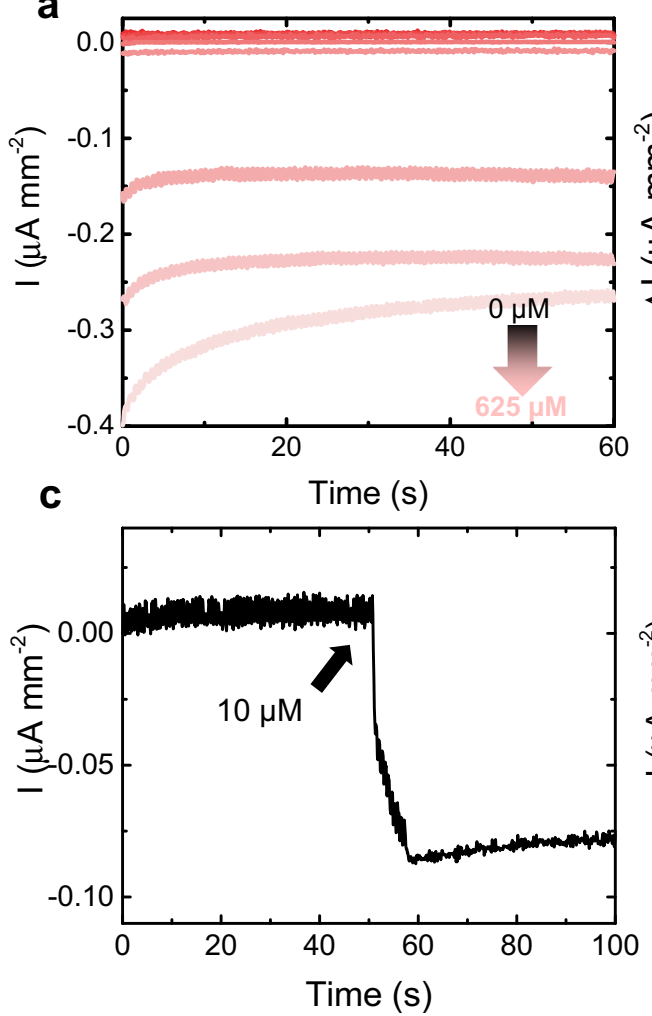

b

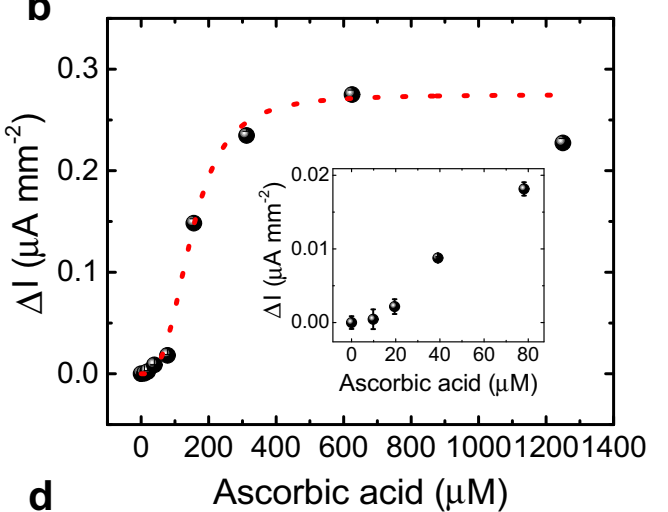

d

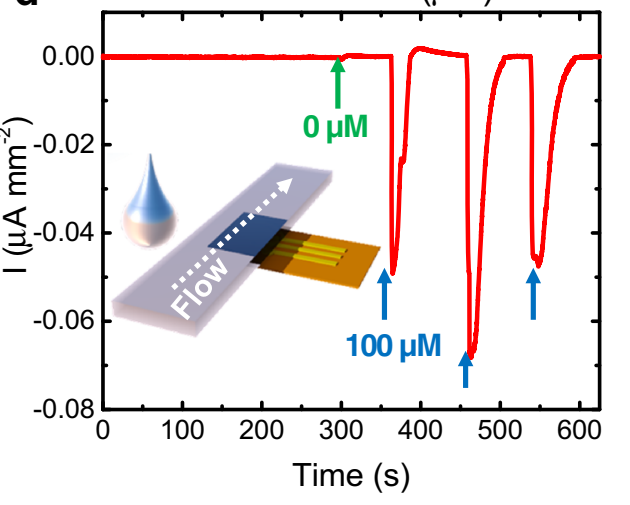




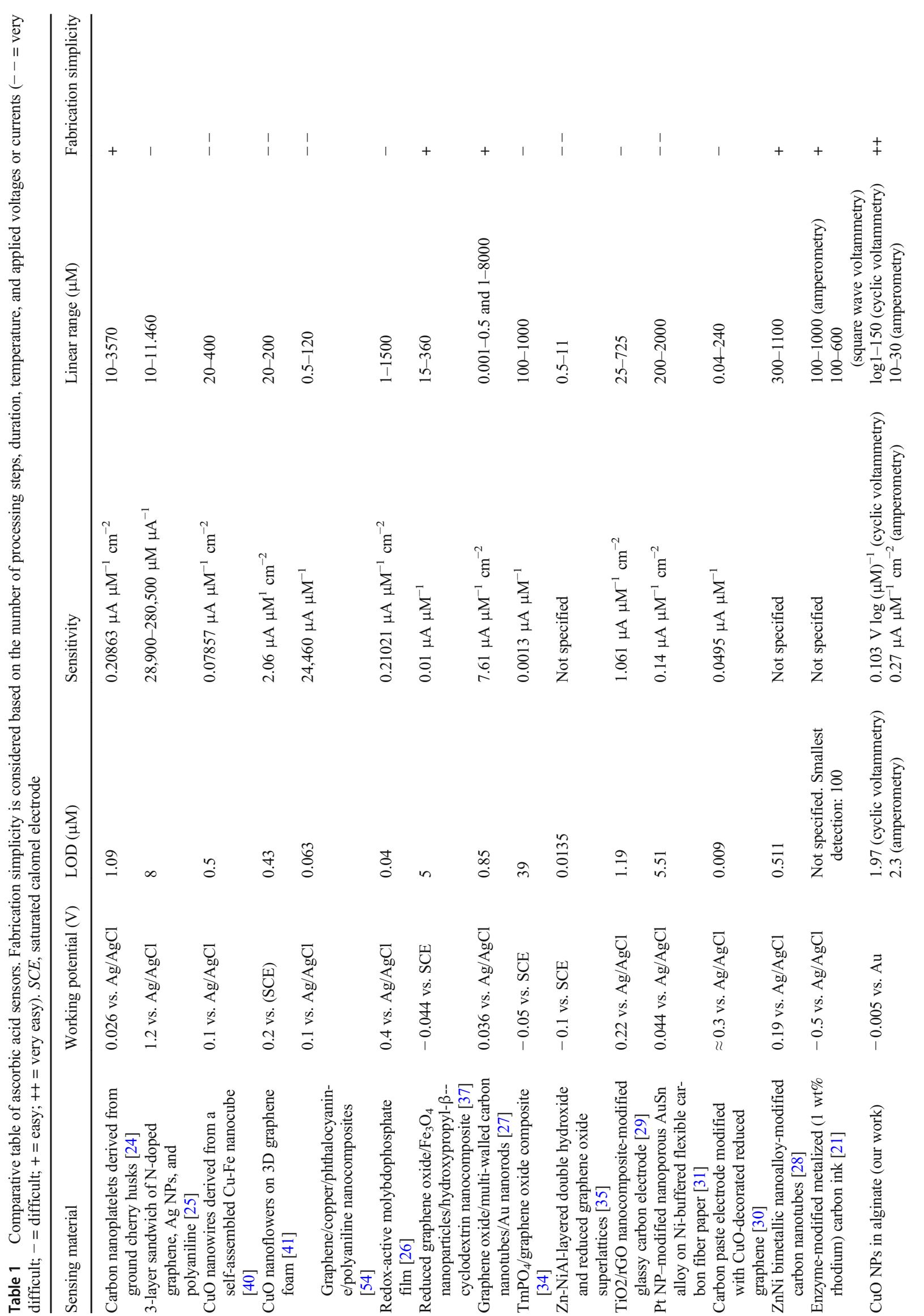


other sensors requiring long processing times of several hours (>24 h) with multiple steps, high temperatures $\left(>350^{\circ} \mathrm{C}\right)$, or high voltage $(>17 \mathrm{~V})$, for nanomaterial synthesis as well as for electrode modification. In addition, our proposed approach performs the measurements in both imidazole (neutral $\mathrm{pH}$ ) and artificial perspiration solution (acidic), convenient for direct determination in human sweat by consuming low power. Alternatively, measurements by cyclic voltammetry are also possible by analyzing the shift of the redox peaks, in order to choose higher current levels where the noise can be smaller. The main limitations of the proposed system, however, come from the aggregate formation of $\mathrm{CuO}$ and the high concentration that is needed despite lower concentrations could already be in molar excess. The concentration is not a major disadvantage considering the low cost of the material, although the reproducibility test shows that $6 \mathrm{mg} \mathrm{mL}^{-1}$ is enough and maintains the same measurable concentration range (unlike $1 \mathrm{mg} \mathrm{mL} \mathrm{m}^{-1}$, which leads to unreproducible results). However, the standard deviation in the resulting current levels makes the device more appropriate for semiquantitative analysis, which can still be used to observe increased amounts of vitamin loss, or absence of it. Regarding the issue of aggregate formation, the addition of a surfactant or solvent that provides a better dispersion of the nanopowder in the alginate precursor without affecting the membrane formation could lead to a better homogeneity of the membrane and the obtained results.

\section{Conclusions}

The hereby demonstrated sensor provides a very simple solution for ascorbic acid determination in sweat, a very convenient fluid for non-invasive physiological parameter monitoring via skin electronics, by combining for the first time the oxidizing capability of $\mathrm{CuO}$ toward the molecule of interest with the facile and quick alginate electrodeposition. Important features of wearable sensors are part of the proposed device, including light weight, determination at ultralow potential vs. Au pseudo-ref. electrode, and compatibility with acidic samples as it is sweat. While both cyclic voltammetry and amperometry are possible, the second approach would allow an easier circuit integration on a wearable device. In addition, the fabrication is done following very few steps and in short time, and commercially available low-cost products are used, which helps to reduce the costs of each final unit. Despite the relatively high concentration of nanoparticles used and the formation of nanoparticle aggregates on the sensing area which could affect reproducibility to some extent, making the system qualitative, future research on the improvement of their dispersion in the precursor solution is a realistic option in order to improve the issue, as aforementioned. Together with the transfer of the technology to electrodes fabricated by screen or inkjet printing, the overall cost could be reduced in the future. Moreover, integration of other catalysts or enzymes for the determination of additional micronutrients, followed by the integration on a wearable electrical device with a miniaturized potentiostat, would enable its implementation in sports or heat-exposed working environments of large duration, protecting the health of the person involved by a timely dietary ascorbic acid intake.

Acknowledgments The authors thank the technical assistance of Dr. Petr Formanek (Leibniz Institute of Polymer Research Dresden e. V.) and Dr. Markus Löffler (Dresden Center for Nanoanalysis, Technical University of Dresden) in the SEM imaging and Dr. Raphael Doineau (Leibniz Institute for Solid State and Materials Research) in the membrane thickness measurement.

Funding information Open Access funding provided by Projekt DEAL. This work was funded by the Arbeitsgemeinschaft industrieller Forschungsvereinigungen (AiF) project F009438.

\section{Compliance with ethical standards}

Conflict of interest The authors declare that there is no conflict of interest.

Open Access This article is licensed under a Creative Commons Attribution 4.0 International License, which permits use, sharing, adaptation, distribution and reproduction in any medium or format, as long as you give appropriate credit to the original author(s) and the source, provide a link to the Creative Commons licence, and indicate if changes were made. The images or other third party material in this article are included in the article's Creative Commons licence, unless indicated otherwise in a credit line to the material. If material is not included in the article's Creative Commons licence and your intended use is not permitted by statutory regulation or exceeds the permitted use, you will need to obtain permission directly from the copyright holder. To view a copy of this licence, visit http://creativecommons.org/licenses/by/4.0/.

\section{References}

1. Ganjali MR et al (2017) Highly sensitive voltammetric sensor for determination of ascorbic acid using graphite screen printed electrode modified with $\mathrm{ZnO} / \mathrm{Al} 2 \mathrm{O} 3$ nanocomposite. Int $\mathrm{J}$ Electrochem Sci 12:3231

2. Lee H, Song C, Hong YS, Kim MS, Cho HR, Kang T, Shin K, Choi SH, Hyeon T, Kim DH (2017) Wearable/disposable sweat-based glucose monitoring device with multistage transdermal drug delivery module. Sci Adv 3:e1601314

3. Tang Y-M et al (2016) Relationships between micronutrient losses in sweat and blood pressure among heat-exposed steelworkers. Ind Health 54:215-223

4. Nováková L, Solich P, Solichová D (2008) HPLC methods for simultaneous determination of ascorbic and dehydroascorbic acids. TrAC Trends Anal Chem 27:942-958

5. Chang YL, Rossetti M, Vlamakis H, Casero D, Sunga G, Harre N, Miller S, Humphries R, Stappenbeck T, Simpson KW, Sartor RB, $\mathrm{Wu}$ G, Lewis J, Bushman F, McGovern DPB, Salzman N, Borneman J, Xavier R, Huttenhower C, Braun J (2019) A screen of Crohn's disease-associated microbial metabolites identifies 
ascorbate as a novel metabolic inhibitor of activated human T cells. Mucosal Immunol 12:457-467

6. Tortajada-Genaro LA (2012) Determination of 1-ascorbic acid in tomato by capillary electrophoresis. J Chem Educ 89:1194-1197

7. Kamiŝalić A, Fister I, Turkanović M, Karakatiĉ S (2018) Sensors and functionalities of non-invasive wrist-wearable devices: a review. Sensors (Switzerland) 18:1714

8. Ibarlucea B, Munoz-Berbel X, Ortiz P, Büttgenbach S, FernándezSánchez C, Llobera A (2016) Self-validating lab-on-a-chip for monitoring enzyme-catalyzed biological reactions. Sensors Actuators B Chem 237:16-23

9. Baraban L, Ibarlucea B, Baek E, Cuniberti G (2019) Hybrid silicon nanowire devices and their functional diversity. Adv Sci 6(15):131. https://doi.org/10.1002/advs.201900522

10. Karnaushenko D, Ibarlucea B, Lee S, Lin G, Baraban L, Pregl S, Melzer M, Makarov D, Weber WM, Mikolajick T, Schmidt OG, Cuniberti G (2015) Light weight and flexible high-performance diagnostic platform. Adv Healthc Mater 4:1517-1525

11. Zhang P et al (2019) Electrochemically exfoliated high-quality $2 \mathrm{H}-$ MoS2 for multiflake thin film flexible biosensors. Small 15: 1901265

12. Miyamoto A, Lee S, Cooray NF, Lee S, Mori M, Matsuhisa N, Jin H, Yoda L, Yokota T, Itoh A, Sekino M, Kawasaki H, Ebihara T, Amagai M, Someya T (2017) Inflammation-free, gas-permeable, lightweight, stretchable on-skin electronics with nanomeshes. Nat Nanotechnol 12:907-913

13. Gao W, Emaminejad S, Nyein HYY, Challa S, Chen K, Peck A, Fahad HM, Ota H, Shiraki H, Kiriya D, Lien DH, Brooks GA, Davis RW, Javey A (2016) Fully integrated wearable sensor arrays for multiplexed in situ perspiration analysis. Nature 529:509-514

14. Koh A et al (2016) A soft, wearable microfluidic device for the capture, storage, and colorimetric sensing of sweat. Sci Transl Med. 8:366ra165

15. Jia W, Bandodkar AJ, Valdés-Ramírez G, Windmiller JR, Yang Z, Ramírez J, Chan G, Wang J (2013) Electrochemical tattoo biosensors for real-time noninvasive lactate monitoring in human perspiration. Anal Chem 85:6553-6560

16. Zamora ML, Dominguez JM, Trujillo RM, Goy CB, Sánchez MA, Madrid RE (2018) Potentiometric textile-based pH sensor. Sensors Actuators B Chem 260:601-608

17. Márquez A, Jiménez-Jorquera C, Domínguez C, Muñoz-Berbel X (2017) Electrodepositable alginate membranes for enzymatic sensors: an amperometric glucose biosensor for whole blood analysis. Biosens Bioelectron 97:136-142

18. Stromberg LR, Hondred JA, Sanborn D, Mendivelso-Perez D, Ramesh S, Rivero IV, Kogot J, Smith E, Gomes C, Claussen JC (2019) Stamped multilayer graphene laminates for disposable infield electrodes: application to electrochemical sensing of hydrogen peroxide and glucose. Microchim Acta 186:533

19. Oh SY, Hong SY, Jeong YR, Yun J, Park H, Jin SW, Lee G, Oh JH, Lee H, Lee SS, Ha JS (2018) Skin-attachable, stretchable electrochemical sweat sensor for glucose and pH detection. ACS Appl Mater Interfaces 10:13729-13740

20. Csiffáry G, Futo P, Adányi N, Kiss A (2016) Ascorbate oxidasebased amperometric biosensor for L-ascorbic acid determination in beverages. Food Technol Biotechnol 54:31-35

21. Sempionatto JR, Khorshed AA, Ahmed A, de Loyola e Silva AN, Barfidokht A, Yin L, Goud KY, Mohamed MA, Bailey E, May J, Aebischer C, Chatelle C, Wang J (2020) Epidermal enzymatic biosensors for sweat vitamin C: toward personalized nutrition. ACS Sensors 5:1804-1813

22. Gnana Kumar G, Amala G, Gowtham SM (2017) Recent advancements, key challenges and solutions in non-enzymatic electrochemical glucose sensors based on graphene platforms. RSC Adv 7: 36949-36976
23. Zhang X, Cao Y, Yu S, Yang F, Xi P (2013) An electrochemical biosensor for ascorbic acid based on carbon-supported PdNi nanoparticles. Biosens Bioelectron 44:183-190

24. Li X, Wang Y, Liu J, Sun M, Bo X, Wang HL, Zhou M (2017) Amperometric ascorbic acid biosensor based on carbon nanoplatelets derived from ground cherry husks. Electrochem Commun 82:139-144

25. Salahandish R, Ghaffarinejad A, Naghib SM, Niyazi A, Majidzadeh-A K, Janmaleki M, Sanati-Nezhad A (2019) Sandwich-structured nanoparticles-grafted functionalized graphene based 3D nanocomposites for high-performance biosensors to detect ascorbic acid biomolecule. Sci Rep 9:1226

26. Liu S, Jiang X, Yang M (2019) Electrochemical sensing of Lascorbic acid by using a glassy carbon electrode modified with a molybdophosphate film. Microchim Acta 186:445

27. Zhao Y, Qin J, Xu H, Gao S, Jiang T, Zhang S, Jin J (2019) Gold nanorods decorated with graphene oxide and multi-walled carbon nanotubes for trace level voltammetric determination of ascorbic acid. Microchim Acta 186:17

28. Savk A, Özdil B, Demirkan B, Nas MS, Calimli MH, Alma MH, Inamuddin, Asiri AM, Șen F (2019) Multiwalled carbon nanotubebased nanosensor for ultrasensitive detection of uric acid, dopamine, and ascorbic acid. Mater Sci Eng C 99:248-254

29. Harraz FA, Faisal M, Ismail AA, al-Sayari SA, al-Salami AE, alHajry A, al-Assiri MS (2019) TiO2/reduced graphene oxide nanocomposite as efficient ascorbic acid amperometric sensor. J Electroanal Chem 832:225-232

30. Karimi-Maleh H, Arotiba OA (2020) Simultaneous determination of cholesterol, ascorbic acid and uric acid as three essential biological compounds at a carbon paste electrode modified with copper oxide decorated reduced graphene oxide nanocomposite and ionic liquid. J Colloid Interface Sci 560:208-212

31. Yang $\mathrm{H}$ et al (2019) Hierarchical bi-continuous Pt decorated nanoporous Au-Sn alloy on carbon fiber paper for ascorbic acid, dopamine and uric acid simultaneous sensing. Biosens Bioelectron 124-125:191-198

32. Wang M, Cui M, Liu W, Liu X (2019) Highly dispersed conductive polypyrrole hydrogels as sensitive sensor for simultaneous determination of ascorbic acid, dopamine and uric acid. J Electroanal Chem 832:174-181

33. Krishnan S, Tong L, Liu S, Xing R (2020) A mesoporous silverdoped $\mathrm{TiO} 2-\mathrm{SnO} 2$ nanocomposite on g-C3N4 nanosheets and decorated with a hierarchical core-shell metal-organic framework for simultaneous voltammetric determination of ascorbic acid, dopamine and uric acid. Microchim Acta 187:82

34. Huang H, Yue Y, Chen Z, Chen Y, Wu S, Liao J, Liu S, Wen HR (2019) Electrochemical sensor based on a nanocomposite prepared from TmPO 4 and graphene oxide for simultaneous voltammetric detection of ascorbic acid, dopamine and uric acid. Microchim Acta 186:189

35. Asif M, Aziz A, Wang H, Wang Z, Wang W, Ajmal M, Xiao F, Chen X, Liu H (2019) Superlattice stacking by hybridizing layered double hydroxide nanosheets with layers of reduced graphene oxide for electrochemical simultaneous determination of dopamine, uric acid and ascorbic acid. Microchim Acta 186:61

36. Zhao Y, Zhou J, Jia Z, Huo D, Liu Q, Zhong D, Hu Y, Yang M, Bian M, Hou C (2019) In-situ growth of gold nanoparticles on a 3D-network consisting of a MoS2/rGO nanocomposite for simultaneous voltammetric determination of ascorbic acid, dopamine and uric acid. Microchim Acta 186:92

37. Liang W, Rong Y, Fan L, Zhang C, Dong W, Li J, Niu J, Yang C, Shuang S, Dong C, Wong WY (2019) Simultaneous electrochemical sensing of serotonin, dopamine and ascorbic acid by using a nanocomposite prepared from reduced graphene oxide, $\mathrm{Fe} 3 \mathrm{O} 4$ and hydroxypropyl- $\beta$-cyclodextrin. Microchim Acta 186:751 
38. George JM, Antony A, Mathew B (2018) Metal oxide nanoparticles in electrochemical sensing and biosensing: a review. Microchim Acta 185:358

39. Cheng X, Zhang X, Yin H, Wang A, Xu Y (2006) Modifier effects on chemical reduction synthesis of nanostructured copper. Appl Surf Sci 253:2727-2732

40. You Q, Liu T, Pang J, Jiang D, Chu Z, Jin W (2019) In situ fabrication of $\mathrm{CuO}$ nanowire film for high-sensitive ascorbic acid recognition. Sensors Actuators B Chem 296(126617):126617

41. Ma Y, Zhao M, Cai B, Wang W, Ye Z, Huang J (2014) 3D graphene foams decorated by $\mathrm{CuO}$ nanoflowers for ultrasensitive ascorbic acid detection. Biosens Bioelectron 59:384-388

42. Ibarlucea B, Roig AP, Belyaev D, Baraban L, Cuniberti G (2019) Single-step modified electrodes for vitamin $\mathrm{C}$ monitoring in sweat. ChemRxiv. https://doi.org/10.26434/CHEMRXIV.11357726.V1

43. Márquez A, Aymerich J, Dei M, Rodríguez-Rodríguez R, VázquezCarrera M, Pizarro-Delgado J, Giménez-Gómez P, Merlos Á, Terés L, Serra-Graells F, Jiménez-Jorquera C, Domínguez C, MuñozBerbel X (2019) Reconfigurable multiplexed point of care system for monitoring type 1 diabetes patients. Biosens Bioelectron 136: $38-46$

44. Mottet L, le Cornec D, Noël JM, Kanoufi F, Delord B, Poulin P, Bibette J, Bremond N (2018) A conductive hydrogel based on alginate and carbon nanotubes for probing microbial electroactivity. Soft Matter 14:1434-1441

45. Cheng Y, Luo X, Betz J, Payne GF, Bentley WE, Rubloff GW (2011) Mechanism of anodic electrodeposition of calcium alginate. Soft Matter 7:5677-5684

46. Kilic T, Brunner V, Audoly L, Carrara S (2017) Smart e-Patch for drugs monitoring in schizophrenia. 2016 IEEE Int Conf Electron Circ Syst ICECS 2016:57-60. https://doi.org/10.1109/ICECS. 2016.7841131
47. Muri H, Hoang L, Hjelme D (2018) Mapping nanoparticles in hydrogels: a comparison of preparation methods for electron microscopy. Appl Sci 8:2446

48. Curto VF et al (2012) Real-time sweat $\mathrm{pH}$ monitoring based on a wearable chemical barcode micro-fluidic platform incorporating ionic liquids. Sensors Actuators B Chem 171-172:1327-1334

49. Coyle, S. et al. (2009) Textile sensors to measure sweat $\mathrm{pH}$ and sweat-rate during exercise. in Proceedings of the $3 \mathrm{~d}$ International ICST Conference on Pervasive Computing Technologies for Healthcare. https:// doi org/10.4108/ICS T. PERVASIVEHEALTH2009.5957

50. Wilde CP, Hu A, Rondeau CM, Wood M (1993) Cyclic voltammetry and charge accumulation at conducting organic salt enzyme electrodes. J Electroanal Chem 353:19-31

51. Schwartz G, Tee BCK, Mei J, Appleton AL, Kim DH, Wang H, Bao Z (2013) Flexible polymer transistors with high pressure sensitivity for application in electronic skin and health monitoring. Nat Commun 4:1859

52. Koczula KM, Gallotta A (2016) Lateral flow assays. Essays Biochem 60:111-120

53. Romeo A, Moya A, Leung TS, Gabriel G, Villa R, Sánchez S (2018) Inkjet printed flexible non-enzymatic glucose sensor for tear fluid analysis. Appl Mater Today 10:133-141

54. Pakapongpan S, Mensing JP, Phokharatkul D, Lomas T, Tuantranont A (2014) Highly selective electrochemical sensor for ascorbic acid based on a novel hybrid graphene-copper phthalocyanine-polyaniline nanocomposites. Electrochim Acta 133:294-301

Publisher's note Springer Nature remains neutral with regard to jurisdictional claims in published maps and institutional affiliations. 